\title{
Quantum correlations in neutrino oscillations in curved spacetime
}

\author{
Khushboo Dixit, ${ }^{1, *}$ Javid Naikoo, ${ }^{1, \dagger}$ Banibrata Mukhopadhyay, ${ }^{2, \ddagger}$ and Subhashish Banerjee ${ }^{1, \S}$ \\ ${ }^{1}$ Indian Institute of Technology Jodhpur, Jodhpur 342011, India \\ ${ }^{2}$ Indian Institute of Science, Bangalore 560012, India
}

(Received 26 March 2019; published 16 September 2019)

\begin{abstract}
Gravity induced neutrino-antineutrino oscillations are studied in the context of one- and two-flavor scenarios. This allows one to investigate the particle-antiparticle correlations in two and four level systems, respectively. Flavor entropy is used to probe the entanglement in the system. The well known witnesses of nonclassicality such as Mermin and Svetlichny inequalities are investigated. Since the extent of neutrinoantineutrino oscillation is governed by the strength of the gravitational field, the behavior of nonclassicality shows interesting features as one varies the strength of the gravitational field. Specifically, the suppression of the entanglement with the increase of the gravitational field is observed which is witnessed in the form of decrease in the flavor entropy of the system. The features of the Mermin and the Svetlichny inequalities allow one to make statements about the degeneracy of neutrino mass eigenstates.
\end{abstract}

DOI: 10.1103/PhysRevD.100.055021

\section{INTRODUCTION}

The phenomenon of neutrino oscillation is well known and establishes the nonzero mass of neutrinos. This idea was first introduced by Pontecorvo to explain the solar neutrino problem and later experimentally confirmed by Super-Kamiokande [1] and Sudbury Neutrino Observatory (SNO) [2]. Till date, several experiments using solar, atmospheric, reactor and accelerator neutrinos have analyzed the data to calculate the oscillation parameters such as mixing angles and mass squared differences. Some of the upcoming experiments are planning to resolve some queries in neutrino sector like, $C P$-violating phase [3], type of mass hierarchy, absolute mass scale of neutrinos [4] and existence of sterile neutrinos.

The effect of gravitational field on neutrino oscillation was also studied [5]. It was shown that while a nonzero (Majorana) mass of neutrinos is required for the neutrinoantineutrino mixing, for oscillation between neutrino and antineutrino (or their mass eigenstates) to occur, their energies must be split. This splitting of energy due to gravitational effect, "gravitational Zeeman effect", also gives rise to the effective charge-parity-time reversal violation, as the effective masses (pertaining to the mass

\footnotetext{
*dixit.1@iitj.ac.in

naikoo.1@iitj.ac.in

tbm@iisc.ac.in

${ }^{\S}$ subhashish@iitj.ac.in
}

Published by the American Physical Society under the terms of the Creative Commons Attribution 4.0 International license. Further distribution of this work must maintain attribution to the author(s) and the published article's title, journal citation, and DOI. Funded by SCOAP . eigenstates) are different [6]. Hence, along with the lepton number violation in the neutrino sector (due to Majorana mass), gravitational Zeeman effect leads to neutrino and antineutrino oscillation. In fact, gravitational Zeeman effect has many other consequences, e.g., neutrino asymmetry, baryogenesis, experimental tests of curvature couplings of spinors, etc., [7-11]. Moreover, the geometric phase in neutrinos has been a part of various studies in this context [12-17]. It is well known that the neutrinos propagating in a varying magnetic field acquire a geometric phase [18]. However, the Zeeman-like splitting was also shown to give rise to a geometric phase where the space-time curvature plays the role of the magnetic field [19].

Gravitational field also modifies the mixing. In absence of gravity, this mixing is passive in the sense that the value of mixing angle is always $\pi / 4$. The effect of gravity leads to active mixing, which depends on the strength of chargeparity-time reversal violation being determined by the strength of gravity itself. The gravity induced neutrinoantineutrino mixing also affects the flavor oscillations, thereby leading to modified neutrino oscillation even in the flavor sector. This naturally invites the investigation of different aspects of neutrino oscillations. In this direction, the present work is devoted to analyzing the neutrino-antineutrino mixing using various tools of quantum foundations.

Among the celebrated notions of quantum foundations are realism and locality. The former says that the existence of an observable quantity does not depend on the observer, while the latter holds that the information cannot reach instantaneously from one point of space to another. These two concepts were used by J. Bell to develop the famous Bell inequity (BI) [20], the violation of which rules out the hypothesis of the hidden variable theory as an alternative of 
quantum mechanics. The Bell type inequalities have been a subject matter of various works, for example, in optical and electronic systems [21-24], in particle physics systems such as mesons [25,26] and neutrinos [27-35]. A time analog of the Bell inequality, known as Legget-Garg inequality has gained a lot of attention recently [36,37]. The various avatars of Bell inequality have been developed to the study of nonlocality in multipartite (more than two) systems, such as Mermin and Svetlichny inequalities. Such manifestation of BI becomes important, for example, when dealing with the three flavors neutrino oscillation.

In this work, we explore various spatial quantum correlations in neutrinos propagating and oscillating in curved spacetimes. We have considered one- and twoflavor neutrino cases, which lead to two- and four-level systems of neutrino-antineutrino oscillation, respectively. To study the correlation measures, the idea of mode entanglement is used [38], as discussed ahead.

The plan of this paper is as follows. In Sec. II we briefly review the gravitational Zeeman effect. This is followed by a discussion of quantum correlations in one- and two-flavor neutrino scenarios in Sec. III. Section IV is devoted to the summary and conclusion.

\section{GRAVITATIONAL “ZEEMAN EFFECT"}

Dirac equation in the presence of background gravitational fields, in a local inertial coordinate, reduces as (see, e.g., $[6,39-41])$

$$
\left[i \gamma^{\mu} \partial_{\mu}-m+i \gamma^{\mu} A_{\mu}^{g}+\gamma^{\mu} \gamma^{5} B_{\mu}^{g}\right] \psi=0,
$$

where $A_{\mu}^{g}$ and $B_{\mu}^{g}$ are the gravitational 4-vector potentials (gravitational coupling with the spinor), $m$ is the mass of spinor, and $\gamma^{5}=\gamma_{5}=i \gamma^{0} \gamma^{1} \gamma^{2} \gamma^{3}$ as usual. Here we choose $\hbar=c=1$. For simplicity, in the rest of the discussion we retain and explore the consequence of the axial-vectorlike term only in equation which suffices for the present purpose. Nevertheless, the vectorlike term might be antiHermitian as well in a local coordinate (depending on the spacetime nature) and can be removed from the total Lagrangian when added to its complex conjugate part. This is particularly so for Majorana neutrinos, when massive neutrinos are most plausibly believed to be Majorana typed. Nevertheless, such a vectorlike antiHermitian term would not contribute to the effective energy of the particle with an appropriate definition of dot-product in curved spacetime [41,42].

Now for the nontrivial solution of $\psi$, the Hamiltonians of the spin-up and spin-down particles are given by

$$
\left(H+\vec{\sigma} \cdot \vec{B}^{g}\right)^{2}=\vec{p}^{2}+B_{0}^{g 2}+m^{2}-2 B_{0}^{g} \vec{\sigma} \cdot \vec{p},
$$

where $B_{0}^{g}$ is the temporal component of $B_{\mu}^{g}$. In the regime of weak gravity and when $m$ is much larger than the rest of the terms in the rhs of Eq. (2), it reduces to

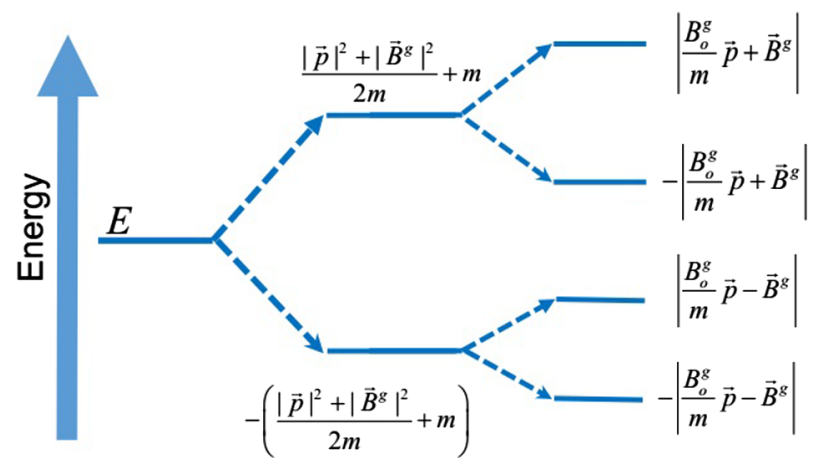

FIG. 1. Gravitational "Zeeman-splitting": Weak gravitational effect, as given by Eq. (3), is considered for the ease of demonstration.

$$
H=-\vec{\sigma} \cdot \vec{B}^{g} \pm\left[\frac{\vec{p}^{2}+B_{0}^{g 2}}{2 m}+m-\frac{B_{0}^{g} \vec{\sigma} \cdot \vec{p}}{m}\right] .
$$

There are two-fold splits in dispersion energy, governed by two terms associated with the Pauli spin matrix, between up and down spinors for positive and negative energy solutions. See Fig. 1 demonstrating the same.

In order to have nonzero $B_{\mu}^{g}$, spherical symmetry has to be broken, hence in Schwarzschild geometry it vanishes. In the Schwarzschild metric, any possible effect would arise from $A_{\mu}^{g}$, which is removed in the present formalism. Indeed it is known $[43,44]$ that spin evolution in spherical symmetric spacetime could arise only from an imaginary Lorentz vectorlike term. On the other hand, in Kerr geometry $B_{\mu}^{g}$ survives. Also it survives in, e.g., early universe under gravity wave perturbation, Bianchi II, VIII, and IX anisotropic universes. Note that in an expanding universe, gravitational potential $B_{\mu}^{g}$ turns to be constant at a given epoch which could act as a background effect.

In the Kerr-Schild coordinate, after putting $\hbar$ and $c$ appropriately, the temporal part of $B_{\mu}^{g}$ reads as

$$
B_{0}^{g}=-\frac{4 a z}{\bar{\rho}^{2} \sqrt{2 r^{3}}} \frac{\hbar c}{r_{g}}
$$

where $\bar{\rho}^{2}=2 r^{2}+a^{2}-x^{2}-y^{2}-z^{2} ; r$ is the radial coordinate of the system expressed in units of $r_{g}, r_{g}=G M / c^{2}$; $M$ and $a$ (varying from -1 to +1 ) are, respectively, mass and dimensionless angular momentum per unit mass of the black hole; $G, c$, and $\hbar$ are, respectively, Newton's gravitation constant, speed of light, and reduced Planck's constant. Naturally, $B_{0}^{g}$ survives (and is varying with space coordinates) for any spinning black hole leading to gravitational Zeeman effect.

In Bianchi II spacetime with, e.g., equal scale-factors in all directions, $B_{0}^{g}$ survives as

$$
B_{0}^{g}=\frac{4+3 y^{2}-2 y}{8+2 y^{2}} \hbar c .
$$




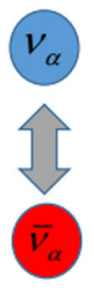

(a)

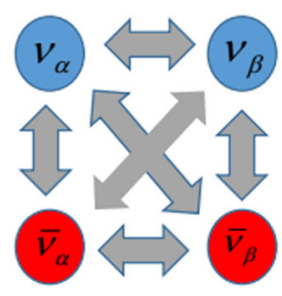

(b)
FIG. 2. Neutrino-antineutrino oscillations in (a) one-flavor, and (b) two-flavor scenarios.

\section{QUANTUM CORRELATIONS IN NEUTRINOS}

We are going to analyze the neutrino-antineutrino oscillations in one- and two-flavor scenarios, which can be viewed as two- and four-level systems, respectively. The schematic diagram is given in Fig. 2.

\section{A. Neutrino-antineutrino mixing in single flavor scenario}

Let us consider, in Weyl representation, a 2-level system describing the mixing of neutrino $(\psi)$ and antineutrino $\left(\psi^{c}\right)$ [45] in the presence of gravitational coupling. We can express the states with known mass (but unknown lepton number states, in the present case spin-states) in terms of states with known lepton/spin-states or vice versa. This is in the spirit of mixing in the neutral kaons, differing by two units of strangeness, whereas for neutrino and antineutrino it differs by two units of lepton number. The corresponding mass eigenstates for a particular flavor at $t=0$ are $[5,6]$

$$
\begin{aligned}
& \left|\nu_{1}(0)\right\rangle=\cos \theta\left|\psi^{c}(0)\right\rangle+e^{i \phi} \sin \theta|\psi(0)\rangle \\
& \left|\nu_{2}(0)\right\rangle=-\sin \theta\left|\psi^{c}(0)\right\rangle+e^{i \phi} \cos \theta|\psi(0)\rangle,
\end{aligned}
$$

when

$$
\tan \theta=\frac{m}{B_{0}^{g}+\sqrt{B_{0}^{g 2}+m^{2}}}, \quad \phi=\arg (-m)
$$

where $m$ is the Majorana mass of the neutrino. Note that the mixing is maximum for $B_{0}^{g}=0$. However, at an arbitrary time $t$ the mass eigenstates are

$$
\begin{aligned}
& \left|\nu_{1}(t)\right\rangle=\cos \theta e^{-i E_{\psi^{c}} t}\left|\psi^{c}(0)\right\rangle+e^{i \phi} \sin \theta e^{-i E_{\psi} t}|\psi(0)\rangle \\
& \left|\nu_{2}(t)\right\rangle=-\sin \theta e^{-i E_{\psi^{c}} t}\left|\psi^{c}(0)\right\rangle+e^{i \phi} \cos \theta e^{-i E_{\psi} t}|\psi(0)\rangle,
\end{aligned}
$$

where the dispersion energies for neutrino and antineutrino, respectively, due to gravitational Zeeman-splitting, from Eq. (2) are given by

$$
\begin{aligned}
E_{\psi} & =\sqrt{\left(\vec{p}-\vec{B}^{g}\right)^{2}+m^{2}}+B_{0}^{g}, \\
E_{\psi^{c}} & =\sqrt{\left(\vec{p}+\vec{B}^{g}\right)^{2}+m^{2}}-B_{0}^{g} .
\end{aligned}
$$

For ultra-relativistic neutrinos, $m \ll|\vec{p}|$ leading to $E_{\psi}-E_{\psi^{c}} \approx 2\left(B_{0}^{g}-\left|\vec{B}^{g}\right|\right)$, the survival probability of $\nu_{1}$ at time $t$ can be expressed as

$$
\mathcal{P}_{s}(t)=1-\sin ^{2} 2 \theta \sin ^{2}\left\{\left(B_{0}^{g}-\left|\overrightarrow{B^{g}}\right|\right)(t)\right\} .
$$

Note that $\left(\nu_{1}(0), \nu_{2}(0)\right)$ is just the transformed spinor of original $\left(\psi^{c}, \psi\right)$. In the limit of zero gravitational effect, i.e., $B_{\mu}^{g} \rightarrow 0, \mathcal{P}_{s}(t) \rightarrow 1$. Thus the neutrino-antineutrino oscillations primarily occur due to nonzero value of the gravitational potential, when the present analysis is performed for ultra-relativistic neutrinos.

In Fig. 3(a), the survival probability for $\nu_{1} \leftrightarrow \nu_{2}$ oscillations is shown as a function of gravitational potential and the distance $\left(L \approx c t\right.$ in ultra relativistic limit $\left.{ }^{1}\right)$ traveled by the neutrino/antineutrino. The survival probability can be seen to approach its maximum value unity as the gravitational potential increases. This implies that gravity suppresses the neutrino-antineutrino oscillations for a fixed $\mathrm{m}$. Further, as noted earlier, the neutrino-antineutrino oscillation approaches maximum when $B_{0}^{g} \rightarrow 0$.

\section{1. von Neumann entropy in oscillation}

The neutrino-antineutrino system can be treated as an effective two qubit system $[29,30,38,46]$ with the following occupation number representation of states defined in Eq. (6) as

$$
\left|\nu_{1}(0)\right\rangle \equiv|10\rangle, \quad\left|\nu_{2}(0)\right\rangle \equiv|01\rangle .
$$

The notation $|10\rangle$ amounts to asking whether we have a $\left|\nu_{1}\right\rangle$ state or not. In this notation, one can finally write

$$
\begin{aligned}
& \left|\nu_{1}(t)\right\rangle=\mathcal{U}_{11}(t)|10\rangle+\mathcal{U}_{12}(t)|01\rangle, \\
& \left|\nu_{2}(t)\right\rangle=\mathcal{U}_{21}(t)|10\rangle+\mathcal{U}_{22}(t)|01\rangle,
\end{aligned}
$$

where the coefficients can be obtained from Eqs. (6) and (8) as

$$
\begin{aligned}
& \mathcal{U}_{11}(t)=\cos ^{2} \theta e^{-i E_{\psi^{c}} t}+\sin ^{2} \theta e^{-i E_{\psi^{\prime}} t}, \\
& \mathcal{U}_{12}(t)=\sin \theta \cos \theta\left(e^{-i E_{\psi} t}-e^{-i E_{\psi^{c}} t}\right), \\
& \mathcal{U}_{21}(t)=\sin \theta \cos \theta\left(e^{-i E_{\psi} t}-e^{-i E_{\psi^{c}} t}\right), \\
& \mathcal{U}_{22}(t)=\sin ^{2} \theta e^{-i E_{\psi^{c}} t}+\cos ^{2} \theta e^{-i E_{\psi} t} .
\end{aligned}
$$

\footnotetext{
${ }^{1}$ Here, entire analysis is performed in local inertial coordinates. Hence, at each point, all the special relativistic norms are conveniently satisfied such that at a given local point, $B_{\mu}^{g}$ appears as constant background field [5].
} 
(a)
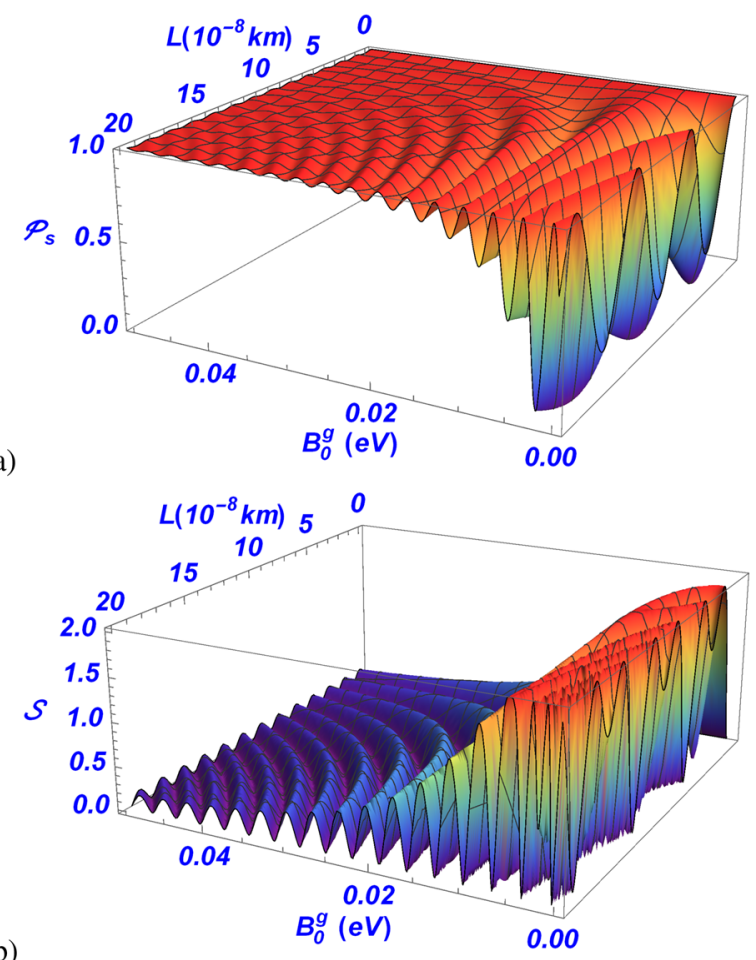

(b)

FIG. 3. Fixed flavor case: (a) Survival probability, and (b) von Neumann entropy, as functions of gravitational potential and distance traveled by neutrino/antineutrino, for the massive states $\nu_{1} \leftrightarrow \nu_{2}$ corresponding to neutrino-antineutrino oscillation. The various parameters used are: $m=5 \times 10^{-3} \mathrm{eV},\left|\overrightarrow{B^{g}}\right| \sim 10^{-2} \mathrm{eV}$.

A standard measure of entanglement for pure states, of form Eq. (11), is given by von Neumann entropy

$$
\mathbf{T}=\left(\begin{array}{cc}
\cos \theta_{e} \cos \phi_{1} & -\cos \theta_{e} \sin \phi_{1} \\
\cos \theta_{\mu} \sin \phi_{1} & \cos \theta_{\mu} \cos \phi_{1} \\
\sin \theta_{e} \cos \phi_{1} & -\sin \theta_{e} \sin \phi_{1} \\
\sin \theta_{\mu} \sin \phi_{1} & \sin \theta_{\mu} \cos \phi_{1}
\end{array}\right.
$$

The mixing angles are related to the masses and the gravitational scalar potential as [5]

$$
\tan \theta_{e, \mu}=\frac{m_{e, \mu}}{B_{0}^{g}+\sqrt{\left(B_{0}^{g}\right)^{2}+m_{e, \mu}^{2}}},
$$

$\tan \phi_{1,2}=\frac{\mp 2 m_{e \mu}}{m_{e(1,2)}-m_{\mu(1,2)}+\sqrt{\left(m_{e(1,2)}-m_{\mu(1,2)}\right)^{2}+4 m_{e \mu}^{2}}}$.

The masses corresponding to the mass eigenstates are given as

$$
\begin{aligned}
\mathcal{S}= & -\sum_{\beta=1,2}\left|\mathcal{U}_{\alpha \beta}(t)\right|^{2} \log _{2}\left|\mathcal{U}_{\alpha \beta}(t)\right|^{2} \\
& -\sum_{\beta=1,2}\left(1-\left|\mathcal{U}_{\alpha \beta}(t)\right|^{2}\right) \log _{2}\left(1-\left|\mathcal{U}_{\alpha \beta}(t)\right|^{2}\right) .
\end{aligned}
$$

Here $\alpha=1(2)$ corresponds to the $\nu_{1}\left(\nu_{2}\right)$ state. For the $\nu_{1} \leftrightarrow \nu_{2}$ oscillation, Fig. 3(b) shows the variation of $\mathcal{S}$ as a function of $B_{0}^{g}$ and $L$. The existence of entanglement is implied by $\mathcal{S}>0$. The von Neumann entropy, as an entanglement measure, is suitable for the neutrino system since it can be expressed in terms of the survival and transition probabilities, which are experimentally measurable quantities [29]. The increase in the gravitational potential is found to decrease the entanglement in the neutrino-antineutrino system for a fixed $\mathrm{m}$. Further, the entropy attains its maximum value when the survival (and hence transition) probabilities, of neutrino and antineutrino, are equal.

\section{B. Two-flavor oscillation with neutrino-antineutrino mixing}

In this case, the flavor and mass eigenstates are related via a unitary matrix $\mathbf{T}$ as follows [5]:

$$
\left(\begin{array}{l}
\psi_{e}^{c} \\
\psi_{\mu}^{c} \\
\psi_{e} \\
\psi_{\mu}
\end{array}\right)=\mathbf{T}\left(\begin{array}{l}
\chi_{1} \\
\chi_{2} \\
\chi_{3} \\
\chi_{4}
\end{array}\right)
$$

where

$$
\left.\begin{array}{cc}
-\sin \theta_{e} \cos \phi_{2} & \sin \theta_{e} \sin \phi_{2} \\
-\sin \theta_{\mu} \sin \phi_{2} & -\sin \theta_{\mu} \cos \phi_{2} \\
\cos \theta_{e} \cos \phi_{2} & -\cos \theta_{e} \sin \phi_{2} \\
\cos \theta_{\mu} \sin \phi_{2} & \cos \theta_{\mu} \cos \phi_{2}
\end{array}\right) .
$$

$$
\begin{aligned}
& M_{1,2}=\frac{1}{2}\left[\left(m_{e 1}+m_{\mu 1}\right) \pm \sqrt{\left(m_{e 1}-m_{\mu 1}\right)^{2}+4 m_{e \mu}^{2}}\right], \\
& M_{3,4}=\frac{1}{2}\left[\left(m_{e 2}+m_{\mu 2}\right) \pm \sqrt{\left(m_{e 2}-m_{\mu 2}\right)^{2}+4 m_{e \mu}^{2}}\right],
\end{aligned}
$$

with

$$
m_{(e, \mu) 1}=-\sqrt{\left(B_{0}^{g}\right)^{2}+m_{e, \mu}^{2}} \quad m_{(e, \mu) 2}=\sqrt{\left(B_{0}^{g}\right)^{2}+m_{e, \mu}^{2}},
$$

and $m_{e \mu}$ being the mixing mass.

Now the system of 2-flavor neutrino oscillations under the influence of neutrino-antineutrino mixing, due to 

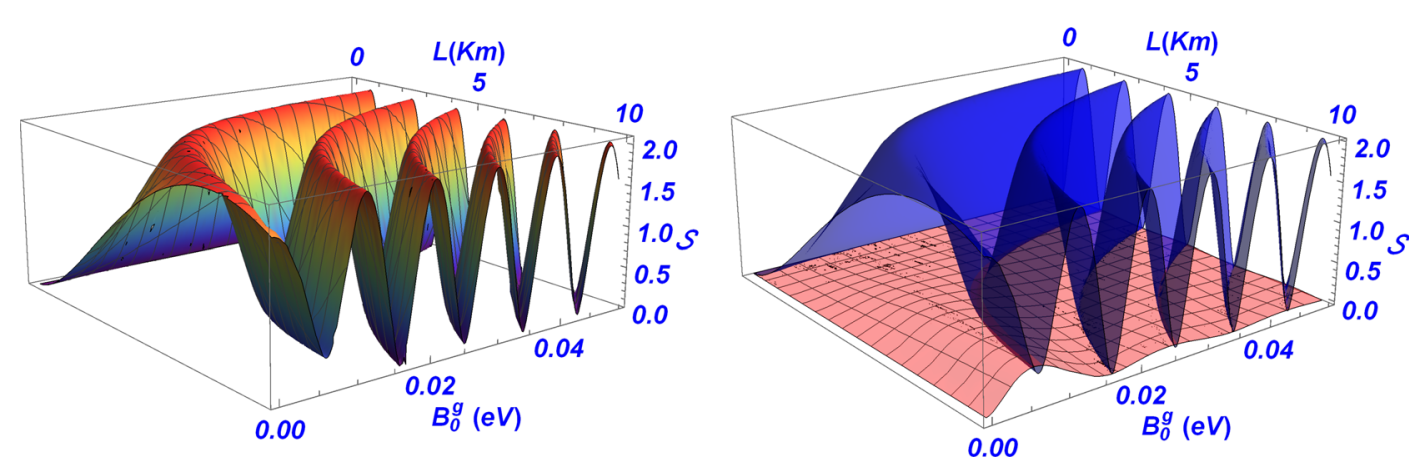

FIG. 4. Left panel: The variation of von Neumann entropy for 2-flavor neutrino-antineutrino oscillation with respect to the distance $(L)$ traveled by neutrinos and the gravitational potential $\left(B_{0}^{g}\right)$. Right panel: The contributions to flavor entropy from neutrino-neutrino oscillations depicted by blue (plane) surface and neutrino-antineutrino oscillations shown by pink (meshed) surface, with the magnitude of $\mathcal{S}$ enhanced 10 times in the later case.

gravitational field, can be treated as a 4-qubit system. The occupation number representation can be given as

$$
\begin{aligned}
\left|\psi_{e}^{c}\right\rangle & \equiv|1\rangle_{\bar{e}} \otimes|0\rangle_{\bar{\mu}} \otimes|0\rangle_{e} \otimes|0\rangle_{\mu}, \\
\left|\psi_{\mu}^{c}\right\rangle & \equiv|0\rangle_{\bar{e}} \otimes|1\rangle_{\bar{\mu}} \otimes|0\rangle_{e} \otimes|0\rangle_{\mu}, \\
\left|\psi_{e}\right\rangle & \equiv|0\rangle_{\bar{e}} \otimes|0\rangle_{\bar{\mu}} \otimes|1\rangle_{e} \otimes|0\rangle_{\mu}, \\
\left|\psi_{\mu}\right\rangle & \equiv|0\rangle_{\bar{e}} \otimes|0\rangle_{\bar{\mu}} \otimes|0\rangle_{e} \otimes|1\rangle_{\mu} .
\end{aligned}
$$

Further, Mermin inequality is a generalized form of Bell inequality and its violation indicates the standard nonlocal correlations existing among different parties in a multipartite system [47]. This means that the probability distribution $P$ (say for a tripartite system) cannot be written in the local form

$P\left(a_{1} a_{2} a_{3}\right)=\int d \lambda \rho(\lambda) P_{1}\left(a_{1} \mid \lambda\right) P_{2}\left(a_{2} \mid \lambda\right) P_{3}\left(a_{3} \mid \lambda\right)$

where $\lambda$ is the shared local variable and $a_{1}, a_{2}, a_{3}$ are the outcomes of the measurements. However, this does not ensure the genuine multipartite nonlocality; i.e., if any two subsystems are nonlocally correlated, but uncorrelated from the third one, Mermin inequality can still be violated $[48,49]$. To probe genuine nonlocal correlations, we make use of the Svetlichny inequality which is based on hybrid nonlocal-local realism [50] as follows:

$P_{B}\left(a_{1} a_{2} a_{3}\right)=\sum_{k=1}^{3} P_{k} \int d \lambda \rho_{i j}(\lambda) P_{i j}\left(a_{i} a_{j} \mid \lambda\right) P_{k}\left(a_{k} \mid \lambda\right)$.

Here the subscript $B$ stands for bipartition sections. For a 4-qubit-system the Mermin $\left(M_{4}\right)$ [51] and Svetlichny $\left(S_{4}\right)$ [52] parameters are defined as

$$
\begin{aligned}
M_{4}= & -A B C D+\left(A B C D^{\prime}+A B C^{\prime} D+A B^{\prime} C D+A^{\prime} B C D\right) \\
& +\left(A B C^{\prime} D^{\prime}+A B^{\prime} C D^{\prime}+A B^{\prime} C^{\prime} D+A^{\prime} B C D^{\prime}\right. \\
& \left.+A^{\prime} B C^{\prime} D+A^{\prime} B^{\prime} C D\right)-\left(A B^{\prime} C^{\prime} D^{\prime}+A^{\prime} B C^{\prime} D^{\prime}\right. \\
& \left.+A^{\prime} B^{\prime} C D^{\prime}+A^{\prime} B^{\prime} C^{\prime} D\right)-A^{\prime} B^{\prime} C^{\prime} D^{\prime}, \\
S_{4}= & A B C^{\prime} D^{\prime}+A B^{\prime} C D^{\prime}+A^{\prime} B C D^{\prime}-A^{\prime} B^{\prime} C^{\prime} D^{\prime} \\
& +A^{\prime} B^{\prime} C D^{\prime}+A^{\prime} B C^{\prime} D^{\prime}+A B^{\prime} C^{\prime} D^{\prime}-A B^{\prime} C^{\prime} D^{\prime} \\
& +A^{\prime} B^{\prime} C D+A^{\prime} B C^{\prime} D+A B^{\prime} C^{\prime} D-A B C D \\
& +A B C^{\prime} D+A B^{\prime} C D+A^{\prime} B C D-A^{\prime} B^{\prime} C^{\prime} D .
\end{aligned}
$$

Here, $X$ and $X^{\prime}(X=A, B, C, D)$ are two different measurement settings pertaining to each qubit. The classical bounds on these parameters are $M_{4} \leq 4$ and $S_{4} \leq 8$. It is important to note that for the violation of Mermin inequality, at least one bipartite section must have the nonlocal correlations, while the Svetlichny inequality will be violated only when all the parties are nonlocally correlated.

In Fig. 4, the variation of von Neumann entropy $(\mathcal{S})$ is depicted with respect to the distance $L$ traveled by neutrinos and the gravitational potential $B_{0}^{g}$. In the twoflavor case the state of the system has four degrees of freedom (d.o.f.) to oscillate between. With initial state as $\nu_{e}$, oscillations occur between $\nu_{e}, \nu_{\mu}, \bar{\nu}_{e}$ and $\bar{\nu}_{\mu}$ flavor modes of the system. Figure 4(a) depicts the total flavor entropy with contribution from all the available modes of oscillation, while in Fig. 4(b) the contribution from particle and antiparticle modes separately is shown. For the sake of clarity, we have enhanced the magnitude of $\mathcal{S}$ by 10 times for the antineutrino case. The particle d.o.f. contribute more to $\mathcal{S}$ in comparison to the antiparticle d.o.f. That is, the neutrino-neutrino flavor mixing is dominating over the neutrino-antineutrino mixing. A common feature depicted in Figs. 3 and 4 is that for neutrino-antineutrino oscillations, $\mathcal{S}$ decreases with the increase in $B_{0}^{g}$. However, for neutrino-neutrino mixing, the increase in $B_{0}^{g}$ does not 

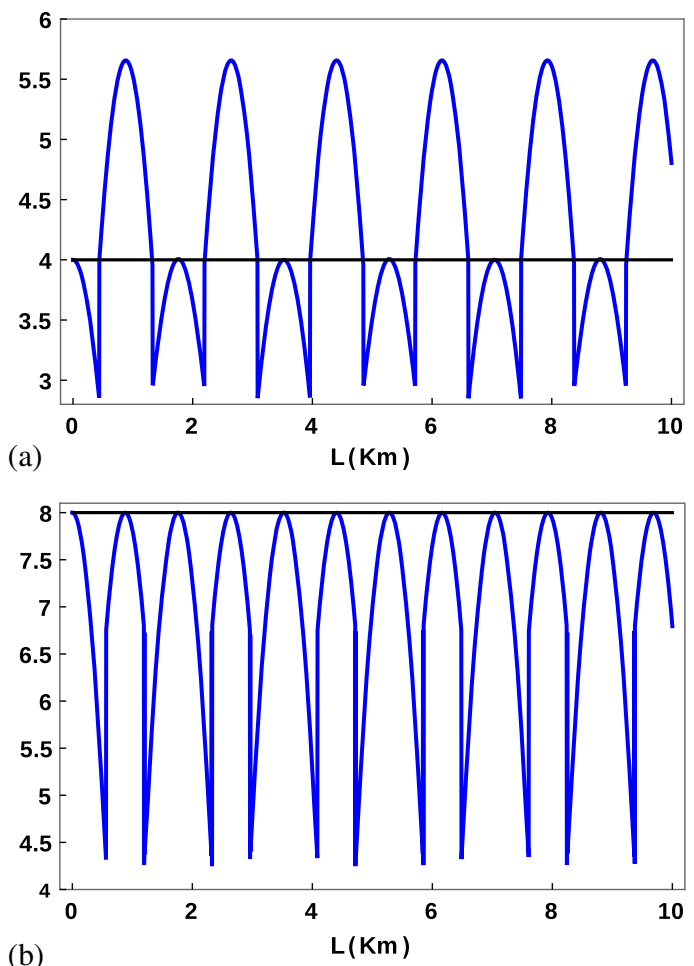

FIG. 5. (a) Mermin $M_{4}$ and (b) Svetlichny $S_{4}$ parameters with respect to the distance $(L)$ traveled by neutrinos with $B_{0}^{g}=5 \times 10^{-2}$. Black lines correspond to the classical bounds of these parameters.

reduce the magnitude of $\mathcal{S}$ but increases the frequency of its oscillation. Figure 5 depicts Mermin and Svetlichny parameters with respect to $L$, with $B_{0}^{g}=5 \times 10^{-2} \mathrm{eV}$. The violation of the classical bound of $M_{4}$ indicates the existence of residual nonlocality in the system. Further, $S_{4}$ does not cross the classical bound in our system, thereby showing the absence of genuine nonlocality. This can be attributed to equality of the two mass squared differences, i.e., $\Delta_{41}=\Delta_{32}=0$, suppressing the nonlocal correlations between the degenerate levels $\nu_{1}-\nu_{4}$ and $\nu_{2}-\nu_{3}$.

\section{SUMMARY AND CONCLUSION}

Spinors interacting with a background gravitational field in an arbitrary spacetime are shown to acquire modified dispersion energy with energies for up and down spinors split: gravitational Zeeman splitting. It has important consequences for neutrino mixing and oscillation and, in general, various quantum correlations. However, for this to have occurred, at least in local coordinates, the spacetime should not be spherical symmetric. To have a nontrivial oscillation phase induced by the gravitational field of, e.g., black holes, such that effects due to neutrino mass are not dominant, the mass of the black hole producing gravitational fields must not be more than a millionth of a solar mass, hence be primordial in nature. In other words, only primordial black holes give rise to any practical gravitational Zeeman splitting. However, without gravitational Zeeman splitting there is no neutrino-antineutrino oscillation even if mixing is nonzero. Moreover, apart from black hole, there are many other scenarios, e.g., early universe particularly in the presence of tensor/gravitational perturbation, where strong gravitational effect leads to neutrino-antineutrino mixing and oscillation.

There are interesting consequences of the interplay between the influence of gravity on neutrino-antineutrino oscillations. Thus, for example, for single-flavor neutrinoantineutrino oscillations, entanglement is maximum for the case when the neutrino and antineutrino states are equally probable. Gravity however suppresses the entanglement between neutrino and antineutrino states, which is implied by a decrease in the von Neumann entropy $\mathcal{S}$ with the increase in the gravitation potential $B_{0}^{g}$.

Further, in case of 2-flavor neutrino-antineutrino oscillations, $\mathcal{S}$ is nonzero which indicates absolute entanglement in the system. Mermin inequality is violated while Svetlichny is not, implying that the system is having absolute nonlocal correlations (nonlocality shared by at least two parties) but genuine nonlocal correlations (nonlocality shared among all parties) are absent, a consequence of the degeneracy in the levels $\nu_{1}-\nu_{4}$ and $\nu_{2}-\nu_{3}$.
[1] S.Fukuda et al.Nucl. Instrum. Methods Phys. Res., Sect. A 501, 418 (2003).

[2] Q. R. e. a. Ahmad (SNO Collaboration), Phys. Rev. Lett. 89, 011301 (2002).

[3] J. Schechter and J. W. F. Valle, Phys. Rev. D 23, 1666 (1981).

[4] I. Y. Kobzarev, B. Martemyanov, L. Okun, and M. Schepkin, Sum rules for neutrino oscillations, Technical Report (Gosudarstvennyj Komitet po Ispol'zovaniyu Atomnoj Ehnergii SSSR, 1981).
[5] M. Sinha and B. Mukhopadhyay, Phys. Rev. D 77, 025003 (2008).

[6] B. Mukhopadhyay, Classical Quantum Gravity 24, 1433 (2007).

[7] S. Mohanty, B. Mukhopadhyay, and A. R. Prasanna, Phys. Rev. D 65, 122001 (2002).

[8] B. Mukhopadhyay, Mod. Phys. Lett. A 20, 2145 (2005).

[9] U. Debnath, B. Mukhopadhyay, and N. Dadhich, Mod. Phys. Lett. A 21, 399 (2006). 
[10] N. E. Mavromatos and S. Sarkar, Eur. Phys. J. C 73, 2359 (2013).

[11] H. J. M. Cuesta, Astrophys. J. 835, 215 (2017).

[12] M. Blasone, P. A. Henning, and G. Vitiello, Phys. Lett. B 466, 262 (1999).

[13] X.-G. He, X.-Q. Li, B. H. J. McKellar, and Y. Zhang, Phys. Rev. D 72, 053012 (2005).

[14] X.-B. Wang, L. C. Kwek, Y. Liu, and C. H. Oh, Phys. Rev. D 63, 053003 (2001).

[15] J. Dajka, J. Syska, and J. Łuczka, Phys. Rev. D 83, 097302 (2011).

[16] P. Mehta, Phys. Rev. D 79, 096013 (2009).

[17] K. Dixit, A. K. Alok, S. Banerjee, and D. Kumar, J. Phys. G 45, 085002 (2018).

[18] S. Joshi and S. R. Jain, Phys. Lett. B 754, 135 (2016).

[19] B. Mukhopadhyay and S. K. Ganguly, arXiv:1802.10377.

[20] J. S. Bell, Phys. Phys. Fiz. 1, 195 (1964).

[21] A. Aspect, P. Grangier, and G. Roger, Phys. Rev. Lett. 47, 460 (1981).

[22] W. Tittel, J. Brendel, B. Gisin, T. Herzog, H. Zbinden, and N. Gisin, Phys. Rev. A 57, 3229 (1998).

[23] B. P. Lanyon, P. Jurcevic, C. Hempel, M. Gessner, V. Vedral, R. Blatt, and C. F. Roos, Phys. Rev. Lett. 111, 100504 (2013).

[24] I. Chakrabarty, S. Banerjee, and N. Siddharth, Quantum Inf. Comput. 11, 0541 (2011).

[25] S. Banerjee, A. K. Alok, and R. MacKenzie, Eur. Phys. J. Plus 131, 129 (2016).

[26] J. Naikoo, A. K. Alok, and S. Banerjee, Phys. Rev. D 97, 053008 (2018).

[27] P. Caban, J. Rembieliński, K. A. Smoliński, Z. Walczak, and M. Włodarczyk, Phys. Lett. A 357, 6 (2006).

[28] A. Bramon, R. Escribano, and G. Garbarino, A review of Bell inequality tests with neutral kaons, in Handbook on Neutral Kaon Interferometry at a $\Phi$-factory, edited by A. Di Domenico, Vol. XLIII (2007), pp. 217-254.

[29] A. K. Alok, S. Banerjee, and S. U. Sankar, Nucl. Phys. B909, 65 (2016).

[30] S. Banerjee, A. K. Alok, R. Srikanth, and B. C. Hiesmayr, Eur. Phys. J. C 75, 487 (2015).
[31] A. Cervera-Lierta, J. Latorre, J. Rojo, and L. Rottoli, SciPost Phys. 3, 036 (2017).

[32] B. Kerbikov, Nucl. Phys. A975, 59 (2018).

[33] Q. Fu and X. Chen, Eur. Phys. J. C 77, 775 (2017).

[34] M. Richter-Laskowska, M. Łobejko, and J. Dajka, New J. Phys. 20, 063040 (2018).

[35] K. Dixit, J. Naikoo, S. Banerjee, and A. K. Alok, Eur. Phys. J. C 78, 914 (2018).

[36] N. Nikitin, V. Sotnikov, and K. Toms, Phys. Rev. D 92 , 016008 (2015).

[37] J. Naikoo, A. K. Alok, S. Banerjee, S. U. Sankar, G. Guarnieri, and B. C. Hiesmayr, arXiv:1710.05562.

[38] M. Blasone, F. Dell'Anno, S. De Siena, and F. Illuminati, Europhys. Lett. 85, 50002 (2009).

[39] N. D. Birrell and P. C. W. Davies, Quantum Fields in Curved Space (Cambridge Univ. Press, Cambridge, England, 1984).

[40] M. Kaku, Quantum Field Theory (Oxford University Press, Oxford, 1993).

[41] J. Schwinger, Particles, Sources, and Fields (AddisonWesley, Reading, MA, 1973).

[42] X. Huang and L. Parker, Phys. Rev. D 79, 024020 (2009).

[43] Y. N. Obukhov, A. J. Silenko, and O. V. Teryaev, Phys. Rev. D 80, 064044 (2009).

[44] M. Dvornikov, Int. J. Mod. Phys. D 15, 1017 (2006).

[45] G. Barenboim, J. F. Beacom, L. Borissov, and B. Kayser, Phys. Lett. B 537, 227 (2002).

[46] M. Blasone, F. Dell'Anno, S. De Siena, and F. Illuminati, Europhys. Lett. 106, 30002 (2014).

[47] N. D. Mermin, Phys. Rev. Lett. 65, 1838 (1990).

[48] D. Collins, N. Gisin, S. Popescu, D. Roberts, and V. Scarani, Phys. Rev. Lett. 88, 170405 (2002).

[49] V. Scarani and N. Gisin, Phys. Rev. Lett. 87, 117901 (2001).

[50] G. Svetlichny, Phys. Rev. D 35, 3066 (1987).

[51] D. Alsina and J. I. Latorre, Phys. Rev. A 94, 012314 (2016).

[52] J.-D. Bancal, N. Brunner, N. Gisin, and Y.-C. Liang, Phys. Rev. Lett. 106, 020405 (2011). 